\title{
Changes in Practice Schedule and Functional Task Difficulty: a Study Using the Probe Reaction Time Technique
}

\author{
Kazunori Akizuki, RPT, MSc ${ }^{1,2)^{*}}$, Yukari Ohashi, RPT, PhD ${ }^{3)}$ \\ 1) Department of Rehabilitation, Misato Central General Hospital: 745 Kobo Misato-city, \\ Saitama 341-8526, Japan. TEL +81 48-953-1321 \\ 2) Graduate School of Health Sciences, Ibaraki Prefectural University of Health Sciences \\ 3) Department of Physical Therapy, Ibaraki Prefectural University of Health Sciences
}

\begin{abstract}
Purpose] Motor learning is accelerated most by optimized task difficulty. When task difficulty is optimized, the amount of information required to complete the task matches the learner's information processing abilities. The practice schedule is one of the factors which changes the amount of task information. We investigated the influence of changes in practice schedule on the amount of task information using the probe reaction time technique. [Methods] Fourteen young male subjects were randomly assigned to a blocked or random practice group. They were required to perform two tasks simultaneously. The primary task consisted of treadmill walking with specific step lengths, and the secondary task consisted of a probe reaction time task. [Results] The blocked practice group was superior to the random practice group in performance during the acquisition phase. In contrast, the random practice group was superior to the blocked practice group in performance during the retention phase. Furthermore, the random practice group had a longer reaction time than the blocked practice group. [Conclusion] From the standpoint of the challenge point framework, motor learning may be accelerated by random practice because random practice probably elicits greater attentional demand than blocked practice.

Key words: Motor learning, Practice schedule, Probe reaction time
\end{abstract}

(This article was submitted Jan. 9, 2013, and was accepted Mar. 8, 2013)

\section{INTRODUCTION}

Practice is considered the most important factor responsible for motor learning. The generalizability of the relationship between practice and skill is so profound that it is sometimes modeled mathematically and referred to as a law ${ }^{1}$. However, in the clinical practice of physical therapy, the practice period is usually limited. Therefore, physical therapists must make the most efficient use of limited opportunities for providing motor learning. Many previous researchers in the motor learning domain have studied the effects of practice variables on motor learning ${ }^{2)}$, especially practice schedule ${ }^{3,4)}$ and the methods for providing feedback $^{5,6)}$.

The most common finding in the extensive literature comparing a blocked practice schedule (e.g., A-A-A, B-B$\mathrm{B}, \mathrm{C}-\mathrm{C}-\mathrm{C}$ ) to a random practice schedule (e.g., A-B-C, B-C$\mathrm{A}, \mathrm{C}-\mathrm{A}-\mathrm{B})$, is that blocked practice produces better performance than random practice during acquisition trials, but that random practice results in better retention performance than blocked practice. This phenomenon is known as the contextual interference effect ${ }^{7}$. However, the majority of previous studies relevant to the contextual interference effect have used relatively simple tasks. Therefore, it has been

*To whom correspondence should be addressed. E-mail: 45030016@ipu.ac.jp suggested that researchers need to study whether contextual interference causes the same phenomenon to occur in complex tasks ${ }^{8}$. Hebert et al. ${ }^{9)}$ studied the relationship between a learner's skill level and contextual interference. In their study, contextual interference was found only in the high-skill group. Albaret and Thon ${ }^{10)}$ reported a contextual interference $\mathrm{x}$ task complexity interaction for tests of transfer. The random practice group was better than the blocked practice group in the transfer tests, but only at the simple task. However, no differences in transfer were found between the random and blocked practice groups at the complex task. Considering these findings, the optimal practice schedule for motor learning is thought to depend, at least, on task difficulty and the learner's skill level.

The challenge point framework has been proposed as explaining the optimal practice conditions changing with the learner's skill level and task complexity ${ }^{11)}$. This framework has two characteristics. First, it indicates that the optimal task difficulty for motor learning differs depending on task complexity, the learner's skill level, and practice conditions. Second, this framework classifies task difficulty into two categories, nominal and functional task difficulties. The nominal difficulty of a task reflects the constant amount of difficulty, regardless of who is performing the task and under what conditions it is being performed. On the other hand, functional task difficulty refers to how challenging the task is relative to the skill level of the individual 
performing the task and to the conditions under which it is being performed. According to the challenge point framework, motor learning will be delayed in the presence of a functional task difficulty which is too high or low. When the amount of information available from performance of the task and the learner's information processing capabilities match, motor learning is most efficient.

However, the influence of the practice schedule on functional task difficulty is still unclear. If the influence were clear, physical therapists would be able to adjust the practice schedule, along with nominal task difficulty and the patients' skill level. In consequence of the adjustment, functional task difficulty would approach the optimal challenge point, and motor learning would become more efficient.

A dual task procedure has been used in many previous studies in order to examine the amount of attention available for performance of a task across a range of perceptual motor tasks ${ }^{12-14)}$. Especially, the probe reaction time (PRT) is a method commonly used for attentional demand measurement during execution of motor tasks ${ }^{15)}$. The attention demand of the primary task is assumed to be inversely related to the PRT; i.e., an extended reaction time is interpreted as indicating that the primary task requires considerable attention.

The purpose of this study was to clarify the effect of the practice schedule on functional task difficulty by measuring functional task difficulty using the PRT.

\section{SUBJECTS AND METHODS}

The subjects of this study were 14 male student volunteers (mean age 21.5 years, $\mathrm{SD}=1.1$ years) who had no prior experience of the experimental tasks and were unaware of the specific purposes of this study. All subjects signed an informed consent before commencing the experiment. Ethical approval for this study was obtained from the Ibaraki Prefectural University of Health Sciences Ethics Committee (Approval No. 398).

Subjects were required to perform two tasks simultaneously. The primary task consisted of walking on a treadmill with a specific length of step. This task required the subjects walk at one of three different lengths of step, $60 \%, 80 \%$, or $100 \%$ of the length of their lower limbs. To calculate the target value of step length, each subject's right lower limb was measured, by a single experimenter, from the anterior superior iliac spine to the apex of the medial malleolus. The subjects were requested to make their steps correspond to the prescribed lengths as much as possible. The treadmill was adjusted to the speed at which the individual subjects felt comfortable. The subjects wore experimental footwear with a pressure-sensitive sensor (PH-462, DKH Co., Ltd.) on the posterior of the heel. At the beginning of a trial, an auditory tone, delivered from a sound simulator, was presented to the subject for one second as the start signal. Then, the subject was told, orally, the length of the target step by the experimenter. The end signal (the same as the start signal) was presented to subject 25 seconds after the start signal. Subjects were instructed not to look down at their feet, but to look straight ahead during execution of the task. A safety bar was mounted on the treadmill to prevent subjects from shifting position on the belt and to maintain their safety. The secondary task consisted of a PRT task, and prior to commencing the experiment, the secondary task was practiced by the subjects sufficiently enough to familiarize themselves with it. Subjects were instructed to respond by saying "pa" as fast as possible upon hearing an auditory tone delivered from a sound simulator. The auditory tones were $50 \mathrm{msec}$ in length and were presented randomly five times with different timings for the acquisition trial. The auditory tones and the subjects' verbal responses were recorded for later analysis.

Subjects were randomly assigned to either a blocked or random practice group; $\mathrm{n}=7$, each group. The experiment was conducted individually in three experimental phases (pretest, acquisition, and posttest) (Fig. 1). After explaining the procedure and task of the experiment to each subject, all the subjects underwent a pretest of 15 trials ( 5 trials for each step length), which were performed in a quasi-random order and did not provide knowledge of results (KR) relative to the difference between the desired value of step length and the obtained value. In this phase, the secondary task was not included. Next, the acquisition phase of 30 trials was administered. During this phase, all the subjects performed the primary and secondary tasks simultaneously. Subjects assigned to the blocked practice condition completed 10 trials of one step length before performing practice trials of a new step length. The practice order of the three step lengths was counterbalanced across subjects. Those individuals assigned to the random practice condition performed 10 trials of each of the three step lengths randomly presented throughout the acquisition phase, with the restriction that one step length was not repeated in consecutive trials. The secondary task stimuli were presented five times at random during the trials. KR of the primary task was orally given to the subjects by the experimenter after every trial. The next trial started after a 10 -second delay. The posttest occurred two to three days later, under the same conditions as the pretest. After the completion of the 15 posttest trials, all groups rested for three minutes. Then the acquisition phase was administered.

Step length $(\mathrm{cm})$ was calculated by multiplying treadmill speed $(\mathrm{cm} / \mathrm{msec})$ by a step time $(\mathrm{msec})$. Step time was defined as the time lapse from one sensor detecting heel contact to the other sensor detecting heel contact. These signals

\begin{tabular}{|c|c|c|c|}
\hline $\begin{array}{c}\text { Day } 1 \\
\text { Pretest } \\
15 \text { Trials } \\
\downarrow \\
\text { Acquisition } \\
30 \text { Trials }\end{array}$ & $\begin{array}{c}\text { Day } 2 \\
\text { Posttest } \\
15 \text { Trials } \\
\downarrow \\
\text { Acquisition } \\
30 \text { Trials }\end{array}$ & $\begin{array}{c}\text { Day } 3 \\
\text { Posttest } \\
15 \text { Trials } \\
\downarrow \\
\text { Acquisition } \\
30 \text { Trials }\end{array}$ & $\begin{array}{c}\text { Day } 4 \\
\text { Posttest } \\
15 \text { Trials }\end{array}$ \\
\hline
\end{tabular}

Fig. 1. Experimental procedure. Pretest reflects the skill level before the acquisition phase. Posttest reflects the results of motor learning in the last acquisition phase (e.g., posttest of Day 2 reflects the results of motor learning in the acquisition phase of Day 1). The time interval between acquisition and the following posttest is $2-3$ days. 
were sent to a dedicated amplifier (PH-450A, DKH Co., Ltd.), and the amplified signals were converted to digital signals using an A/D converter (Power Lab/16SP; AD Instruments) at a sampling rate of $100 \mathrm{~Hz}$ and stored a personal computer. The step length error was the absolute value of the difference between the desired step length and the obtained step length divided by the length of the individual's lower limb. PRT greater than two standard deviations above or below the mean were removed as outliers. To obtain the rate of prolongation of PRT caused by performance of the primary task, the PRT when performing the primary task was divided by the PRT when walking on the treadmill at the usual step length of individual subjects.

SPSS ver. 20 software (SPSS Statistics, IBM Corp) was used to perform the statistical analysis. The error of step length in the acquisition phase and PRT were analyzed in a 2 (practice schedule conditions) $\times 3$ (Day) ANOVA, with repeated measures for the last factor. The error of step length in all test phases were analyzed in a 2 (practice schedule conditions) $\times 4$ (Day) ANOVA, with repeated measures for the last factor. We chose an alpha level of 0.05 to indicate significant effects.

\section{RESULTS}

The error of step length of each of the two groups during the acquisition phase is shown in Table 1. Both groups demonstrated a consistent decrease in the error of the step length across days. The main effects of day and practice schedule were significant. In addition to these main effects, day $\times$ practice schedule interaction was significant. To fur- ther investigate this interaction, the independent sample t-test was used to test the significance of the difference between groups on each day. The results show that the blocked practice group made significantly less error than the random practice group on all days (Day $1, \mathrm{p}<0.05$; Day 2, $\mathrm{p}<$ 0.05; Day 3, $\mathrm{p}<0.05$ ).

The error of step length of each of the two groups during the test phase is shown in Table 2. Both groups demonstrated a consistent decrease in the error of the step length across days. The main effects of day and practice schedule were significant. In addition to these main effects, day $\times$ practice schedule interaction was significant. To further investigate this interaction, the independent sample t-test was used to test the significance of the difference between groups on each day. The results show that although there was no significant difference between the blocked and random practice groups at the pretest, the random practice group made significantly fewer errors than the random practice group on all posttests (Day 2, p $<0.05$; Day 3, p $<0.05$; Day 4, p $<0.05)$.

The PRT of each of the two groups during the acquisition phase is shown in Table 3. The analysis revealed that there was a significant decrease in PRT across the acquisition phase. The main effects of day and practice schedule were significant. In addition to these main effects, day $\times$ practice schedule interaction was significant. To further investigate this interaction, the independent sample t-test was used to test the significance of the difference between groups on each day. Although there was no significant difference between the blocked and random practice groups on Day 1, the random practice group had a significantly longer PRT

Table 1. The error of step length of each of the two groups during the acquisition phase

\begin{tabular}{lccc}
\hline & \multicolumn{3}{c}{ Acquisition phase } \\
\cline { 2 - 4 } & Day $1^{*}$ & Day 2* & Day 3* \\
\hline Blocked practice & $4.2 \pm 4.3$ & $2.5 \pm 2.7$ & $2.1 \pm 2.1$ \\
Random practice & $7.8 \pm 7.1$ & $3.6 \pm 3.1$ & $3.1 \pm 2.8$ \\
\hline Mean \pm SD (\%). *: significant difference between blocked and random practice groups (p \\
$<0.05)$
\end{tabular}

Table 2. The error of step length of each of the two groups during the test phase

\begin{tabular}{lcccc}
\hline & Pretest & \multicolumn{3}{c}{ Posttest } \\
\cline { 3 - 5 } & & Day 2* & Day 3* & Day 4* \\
\hline Blocked practice & $15.1 \pm 9.1$ & $10.5 \pm 7.2$ & $6.7 \pm 4.4$ & $5.0 \pm 3.6$ \\
Random practice & $16.8 \pm 12.2$ & $7.3 \pm 6.8$ & $5.4 \pm 3.7$ & $4.0 \pm 2.9$ \\
\hline
\end{tabular}

Mean \pm SD (\%). *: significant difference between blocked practice and random practice $(\mathrm{p}<0.05)$

Table 3. The PRT of each of the two groups during the acquisition phase

\begin{tabular}{lccc}
\hline & \multicolumn{3}{c}{ Acquisition phase } \\
\cline { 2 - 4 } & Day 1 & Day 2* & Day 3* \\
\hline Blocked practice & $133.8 \pm 27.2$ & $125.0 \pm 23.2$ & $121.7 \pm 23.3$ \\
Random practice & $133.9 \pm 26.1$ & $127.1 \pm 25.1$ & $126.6 \pm 26.1$ \\
\hline Mean \pm SD (\%). *: significant difference between blocked practice and random practice \\
$(\mathrm{p}<0.05)$
\end{tabular}


than the blocked practice group on Day 2 and Day 3 (Day 2, $\mathrm{p}<0.05$; Day 3, $\mathrm{p}<0.05)$.

\section{DISCUSSION}

Functional task difficulty that affects the efficiency of motor learning consists of task complexity, the learner's skill level, and practice condition. In this study, the influence on the functional task difficulty of difference in skill level was excluded by setting a novel task (one subjects had not yet learned), and by verifying that there was no difference in the skill level of each group at the time of the pretest. In addition, because all subjects executed the same tasks, the study protocol ensured that no difference existed in the nominal task difficulty between the groups. Therefore, the difference of the functional task difficulty for each group was attributable to the different practice schedules. This study examined the influence on functional task difficulty of the practice schedule using the PRT technique. The blocked practice group showed significantly superior performance to the random practice group during the acquisition phase. In contrast, the random practice group showed significantly superior performance to the blocked practice group during the retention phase. This result is in agreement with those of many previous studies concerning practice schedule and provides further evidence in support of the contextual interference effect.

Several hypotheses have been proposed for the contextual interference effect. The two most prominent hypotheses center on the benefits of encoding ${ }^{7,16)}$, and the benefits resulting from forgetting and subsequent reconstruction ${ }^{17)}$. According to Shea and Zimny ${ }^{16)}$, the difference in task requirements during random practice facilitates better comparative and contrastive analyses of the features of different tasks, resulting in the formation of a more elaborate and distinct memory representation. This means that the random practice group would have developed a memory representation that facilitates retrieval. The other hypothesis ${ }^{17)}$ is based on ideas about the spacing effect originally presented in verbal learning literature. According to this hypothesis, the action planning that occurs just prior to a practice trial is influenced by what was done in the previous trial. In blocked practice, a previously constructed action plan is available in working memory because the same task has just been performed, and no forgetting occurs. In contrast, when tasks are ordered intermittently during random practice, the previously constructed action plan must be abandoned to execute other tasks and then reconstructed once again. This view accounts for the learning/performance distinction of the contextual interference effect, because remembering the action from a previous trial (as in blocked practice) facilitates better performance in acquisition, but does not facilitate learning. However, random practice creates short term retention loss, which is detrimental to acquisition performance, but beneficial to learning.

The important thing to understand here is that increased cognitive efforts attributable to random practice inhibit performance during acquisition, but facilitate learning in both hypotheses. We consider that the increased cognitive efforts attributable to random practice represent increased functional task difficulty. Our results revealed that the random practice resulted in a longer PRT than the blocked practice, suggesting the functional task difficulty of the random practice is higher than the functional task difficulty of the blocked practice. This further suggests that the functional task difficulty of random practice rises due to the contextual interference effect. As the functional task difficulty was increased, performance during the acquisition period was inhibited, but motor learning was facilitated because the increased functional task difficulty approached the optimal challenge point. Our results indicate that practice schedule affects the functional task difficulty, and increased functional task difficulty makes motor learning more efficient. Extending these results to the clinical settings of physical therapy, motor learning would be more efficient if random practice were adopted in cases where the learning task is too easy. Similarly, motor learning would be more efficient if blocked practice were adopted in cases where the learning task is too difficult.

We recognize several limitations of the study. First, it is important to point out that all the subjects were young men, and the results may be affected by age and gender. Second, this study used treadmill walking with specific step lengths as the learning task; therefore, the generalization of results to other tasks may be limited. Third, we failed to confirm the relationship between the functional task difficulty and the efficiency of motor learning when the functional task difficulty exceeds the optimal challenge point. Therefore, in future studies, we should consider the relationship between the functional task difficulty and efficiency of motor learning when it exceeds the optimal challenge point.

\section{REFERENCES}

1) Crossman E: A theory of the acquisition of speed-skill. Ergonomics, 1959, 2: 153-166. [CrossRef]

2) Adams JA: Historical review and appraisal of research on the learning, retention, and transfer of human motor skills. Psychol Bull, 1987, 101: 41-74. [CrossRef]

3) Magill RA, Hall KG: A review of the contextual interference effect in motor skill acquisition. Hum Mov Sci, 1990, 9: 241-289. [CrossRef]

4) Brady F: A theoretical and empirical review of the contextual interference effect and the learning of motor skills. Quest, 1998, 50: 266-293. [CrossRef]

5) Schmidt RA: Frequent Augmented Feedback can Degrade Learning: Evidence and Interpretations. In: Requin J and Stelmach GE (eds.): Tutorials in Motor Neuroscience. Dordrecht: Kluwer Academic Publishers, 1991, pp $59-75$.

6) Swinnen SP: Information Feedback for Motor Skill Learning: A Review. In: Zelaznik HN (ed.): Advances in motor learning and control. Champaign: Human Kinetics, 1996, pp 37-66.

7) Shea JB, Morgan RL: Contextual interference effects on the acquisition, retention, and transfer of a motor skill. J Exp Psychol Hum Learn, 1979, 5: 179-187. [CrossRef]

8) Wulf G, Shea CH: Principles derived from the study of simple skills do not generalize to complex skill learning. Psychon Bull Rev, 2002, 9: 185-211. [Medline] [CrossRef]

9) Hebert EP, Landin D, Solmon MA: Practice schedule effects on the performance and learning of low- and high-skilled students: an applied study. Res Q Exerc Sport, 1996, 67: 52-58. [Medline] [CrossRef]

10) Albaret JM, Thon B: Differential effects of task complexity on contextual interference in a drawing task. Acta Psychol (Amst), 1998, 100: 9-24. [Medline] [CrossRef]

11) Guadagnoli MA, Lee TD: Challenge point: a framework for conceptualizing the effects of various practice conditions in motor learning. J Mot 
Behav, 2004, 36: 212-224. [Medline] [CrossRef]

12) Ells JG: Analysis of temporal and attentional aspect of movement control. J Exp Psychol, 1973, 99: 10-21. [Medline] [CrossRef]

13) Kerr B: Processing demands during movement. J Mot Behav, 1975, 7 $15-27$.

14) Glencross DJ, Gould JH: The planning of precision movement. J Mot Behav, 1979, 11: 1-9. [Medline]
15) Salmoni AW, Sullivan SJ, Starkes JL: The attention demands of movements: a critique of the probe technique. J Mot Behav, 1976, 8: 161-169.

16) Shea JB, Zimny ST: Context Effects in Learning Movement Information. In: Magill RA (ed.): Memory and the control of action. Amsterdam: NorthHolland, 1983, pp 345-366.

17) Lee TD, Magill RA: The locus of contextual interference in motor-skill acquisition. J Exp Psychol Learn Mem Cogn, 1983, 9: 730-746. [CrossRef] 